\title{
ON THE BOUNDEDNESS OF SOLUTIONS OF NONLINEAR DIFFERENTIAL AND DIFFERENCE EQUATIONS
}

\author{
BY \\ RICHARD BELLMAN
}

1. Introduction. In this paper we wish to investigate the nature and existence of bounded solutions of systems of differential equations of the form

$$
\begin{array}{r}
d z_{i} / d t=\sum_{j=1}^{N} a_{i j}(t) z_{j}+f_{i}\left(z_{1}, z_{2}, \cdots, z_{N}, d z_{1} / d t, d z_{2} / d t, \cdots, d z_{N} / d t, t\right) \\
i=1, \cdots, N .
\end{array}
$$

The variables $z_{1}, z_{2}, \cdots, z_{N}$ are real, and $t$ ranges over the interval $(0, \infty)$. The $f_{i}$ will be subject to various conditions, but all will share the common property that

$$
\left|f_{i}\left(z_{1}, z_{2}, \cdots, z_{N}, w_{1} w_{2}, \cdots, w_{N}, t\right)\right|=o\left(\sum_{k=1}^{N}\left|z_{k}\right|+\left|w_{k}\right|\right)
$$

as $\sum_{k=1}^{N}\left|z_{k}\right|+\left|w_{k}\right| \rightarrow 0$, for fixed $t$.

Systems of the form (1.1) are of considerable interest in dynamics, and play an important role in many branches of applied mathematics. Usually the right-hand side does not involve any derivatives. In dynamics, where $t$ represents the time, a natural problem is the determination of the behavior of the solutions for large values of the time, and this is the central problem considered in the paper. The behavior of the solution turns out to depend critically upon the initial values, and thus the question becomes one of stability in the sense of Liapounoff.

A solution, $s$, is said to be stable in the sense of Liapounoff if every solution, $s^{\prime}$, whose initial values are "close" to those of $s$ remains "close" to $s$ for all subsequent values of $t$. The word "close" is defined by a suitable metric. If the two solutions are given by $z_{i}, z_{i}^{\prime}, i=1,2, \cdots, N$, the distance between them will be taken to be $\sum_{i=1}^{N}\left|z_{i}-z_{i}^{\prime}\right|$. In our case, since $f_{i}(0,0, \cdots, 0, t)$ $=0, z_{i}=0, i=1,2, \cdots, N$, is a solution of (1.1). Letting $a_{i}=z_{i}(0)$ be the initial values of any other solution of (1.1), we shall show that provided that $\sum_{i=1}^{N}\left|a_{i}\right|$ is sufficiently small this solution remains small for all $t>0$.

This investigation, for the case where the $f_{i}$ are power series in the $z_{k}$ beginning with second degree terms, and the $a_{i j}$ are constants, was initiated by Poincaré, and pursued extensively by Liapounoff. Subsequent researches

Presented to the Society, April 26, 1947; received by the editors November 5, 1946. 
are due to Bohl, Cotton, and Perron $[10,11]\left({ }^{1}\right)$, where references are given. Perron [10] obtained the following precise result:

TheOrem 1 (PERron). Consider the following system of differential equations

$$
d z_{i} / d t=\sum_{j=1}^{N} a_{i j} z_{j}+f_{i}\left(z_{1}, z_{2}, \cdots, z_{N}, t\right), \quad i=1,2, \cdots, N
$$

where

(1.4) (1) All the characteristic roots of the constant matrix $A=\left(a_{i j}\right)$ have negative real parts,

(2) $\left|f_{i}\left(z_{1}, z_{2}, \cdots, z_{N}, t\right)\right|=o\left(\sum_{k=1}^{N}\left|z_{k}\right|\right)$ as $\sum_{k=1}^{N}\left|z_{k}\right| \rightarrow 0$, uniformly in $t$.

Under these conditions, any solution of (1.3) whose initial values are sufficiently close to the zero solution is uniformly bounded for all $t$, and tends to the zero solution as $t$ approaches infinity.

Perron's result is very elegant and satisfying as to the qualitative behavior of the solutions, but does not furnish quantitative information. The method of proof used by Perron does not require that one actually exhibit any particular solution, or discuss its behavior for large $t$. While the standard existence theorems ensure the existence of solutions of (1.3) under reasonable conditions on the $f_{i}$, the form of the solution obtained in the course of the proofs seems clearly unsuited to the determination of its asymptotic behavior, even though we are forearmed, by means of Theorem 1, with the knowledge that it must tend to the zero solution as $t \rightarrow+\infty$.

In this paper we intend to generalize Theorem 1 to cover equations of the form of (1.1), and to state corresponding results for the case where all the characteristic roots of the matrix $A=\left(a_{i j}\right)$ merely have nonpositive real parts. Also, we shall discuss the situation where $A$ is a variable matrix. Perron [11] also considered this problem and obtained results under conditions upon $A$ different from ours. Here the results are necessarily more fragmentary.

Several methods will be used, two depending upon explicit representation of the solution as the limit of a sequence, and thus available for numerical calculation, and one merely guaranteeing the existence of solutions of the desired type. Both methods involving sequences require the existence of the partial derivatives of $f_{i}$ with respect to $z_{1}, z_{2}, \cdots, z_{N}, d z_{1} / d t, d z_{2} / d t, \cdots$, $d z_{N} / d t$, and then conditions' on these derivatives. However, for the equation in (1.3) one method dispenses with these restrictions.

In a written communication to the author, Levinson has stated that the

(1) Numbers in brackets refer to the references cited at the end of the paper. 
result corresponding to Theorem 1 will hold for the equation of (1.1) also. But again his method of proof is similar to Perron's in that it does not furnish any hold on the quantitative behavior of the function for large $t$. The advantage of the methods used in this paper lies in this ability to represent the solution in a form exhibiting its behavior.

In the second part of the paper, we consider difference equations of the type

$$
\begin{aligned}
& z_{i}(t+1)=\sum_{j=1}^{N} a_{i j}(t) z_{j}(t) \\
& +f_{i}\left(z_{1}(t), \cdots, z_{N}(t), z_{1}(t+1), \cdots, z_{N}(t+1), t\right), \\
& \quad i=1,2, \cdots, N ; t=0,1, \cdots .
\end{aligned}
$$

Ta $\mathrm{Li}$ [14] and Perron himself [12] applied the methods of Perron to obtain results for difference equations of the above class similar to Theorem 1. In treating (1.5), our method developed for differential equations seems much simpler than that of Ta Li's, and again has the advantage of illustrating the behavior of the solution in direct fashion. In addition to the analogue of Theorem 1, we derive some new results.

In the first part of the paper we introduce the principal method of the paper, the conversion of the differential equation into a suitable integral equation. This method originated with Liouville and has furnished a very powerful means of obtaining the asymptotic behavior of the eigenfunctions and eigenvalues of differential equations. This method was used by the author [1], Levinson [7], and Weyl [15], to discuss the stability of linear differential systems. The integral equations used to prove existence theorems of the ordinary type are much weaker instruments of research when it comes to determining asymptotic behavior.

We shall now sketch the method. Let $y$ denote a column vector whose components are $y_{1}, y_{2}, \cdots, y_{N}$. Then the equation of the first approximation

$$
d y_{i} / d t=\sum_{j=1}^{N} a_{i j} y_{i}, \quad i=1,2, \cdots, N,
$$

can be written more simply as

$$
d y / d t=A y
$$

where $A$ is the coefficient matrix $\left(a_{i j}(t)\right)$. Similarly, (1.1) can be written

$$
d z / d t=A z+f(z, d z / d t, t)
$$

where $z$ and $f(z, d z / d t, t)$ are respectively column vectors with the components $z_{i}$ and $f_{i}$. Now let $Y$ be the $N \times N$ matrix satisfying the equation

$$
d Y / d t=A Y, \quad Y(0)=\cdot I .
$$


Every column of $Y$ satisfies (1.6). It is now easy to show that the solution of (1.8) satisfying the boundary conditions $z\left(t_{0}\right)=y\left(t_{0}\right), y(t)$ satisfying (1.7), is a solution of the integral equation

$$
z(t)=y(t)+\int_{t_{0}}^{t} Y(t) Y^{-1}\left(t_{1}\right) f\left(z, d z / d t_{1}, t_{1}\right) d t_{1} .
$$

The method of successive approximations is now used to show that a solution of (1.10) exists and possesses the desired properties, under various hypotheses on $A$ and $f(z, d z / d t, t)$. The sequence defined by recurrence

$$
z_{0}=y,
$$

$$
z_{n+1}=y+\int_{t_{0}}^{t} Y(t) Y^{-1}\left(t_{1}\right) f\left(z_{n}, d z_{n} / d t_{1}, t_{1}\right) d t_{1}, \quad n \geqq 0,
$$

is shown to converge uniformly. In this way, the classical results of PoincaréLiapounoff concerning nonlinear systems are obtained together with more recent results of Hukuwara concerning linear systems.

Equation (1.10) furnishes a link between the solutions of (1.6) and (1.8) which suffices in the majority of cases to exhibit the connection between the behaviors at infinity of the solutions of the two equations.

The method of successive approximations is not powerful enough in some cases. To treat these cases, two other methods are available. When $d z / d t$ does not occur on the right-hand side of (1.1) an extension of the BirkhoffKellogg fixed-point theorem, due to Hukuwara [6], can be used. Regarding (1.8) as an equation of the form $z=T(z)$, it is easy to verify that $T(z)$ satisfies the conditions of the theorem. While the method fails to exhibit the solution, and generally yields no information as to uniqueness (except for one case where it can be combined with the standard existence theorem to yield uniqueness), it seems worthwhile to include it, since it furnishes an interesting proof of existence and boundedness simultaneously. The second method will be discussed later.

In the second part of the paper the difference equations

$$
\Delta z=z(t+1)-z(t)=A z(t)+f(z(t), z(t+1), t), \quad t=0,1, \cdots,
$$

are discussed by means of the recurrence relation

$$
z(t)=y(t)+\sum_{k=t}^{t-1} Y(t) Y^{-1}(k+1) f(z(k), z(k+1), k)
$$

connecting the solutions of

$$
\Delta y=A y
$$

and those of (1.12). $Y$ is a matrix analogous to that defined for differential equations. 
The theory is considerably simpler for difference equations when $z(t+1)$ does not appear on the right-hand side. Since $z(t+1)$ is given in terms of $z(\bar{k})$, $t_{0} \leqq k \leqq t-1$, induction can be effectively used. If $z(t+1)$ does appear, we use the method of successive approximations again.

Applying the results obtained for difference equations, we shall sketch the second method applicable to differential equations, a method which requires no stronger restrictions than those imposed by the fixed-point methods, and yet furnishes a constructive proof of the existence, and may be used for calculations. Approximate (1.1) (considering only $z$ and $t$ appearing on the right-hand side) by the difference equation

$$
z(t+h)-z(t)=h A z(t)+h f(z, t) .
$$

The theorems obtained for difference equations show that for $h$ small the solutions of (1.15) are bounded for all $t, t=0, h, 2 h, \cdots$. Functions defined for all $t$ can be obtained by defining their values to be linear in between these points. It is easy to show that these functions are equicontinuous, and if we consider any finite interval, Arzela's selection theorem can be used. It is true that this method only proves the existence of a convergent sequence, which is a subsequence of a larger sequence, but practically, convergence could always be recognized numerically.

The methods described above have proved applicable to a large class of nonlinear partial differential equations, of which the following is one type

$$
\frac{\partial^{2} u}{\partial x^{2}}+\frac{\partial^{2} u}{\partial y^{2}}+\frac{\partial^{2} u}{\partial z^{2}}-\frac{\partial u}{\partial t}=F(u, x, y, z, t)
$$

under various types of boundary conditions restricting the space variables, $x, y, z$, to a finite region, and $t$ to the semi-infinite interval $(0, \infty)$.

We hope to discuss these results in a subsequent paper.

The author wishes to take this opportunity to express his appreciation to Professor Lefschetz who first introduced him to the researches of Poincaré and Liapounoff, and whose continued interest in the topic of the paper, and many conversations with the author, have proved a source of great stimulation.

\section{Part I. Differential equations}

2. Preliminary lemmas. Consider the differential equations

$$
\begin{array}{rlr}
d z_{i} / d t=\sum_{j=1}^{N} a_{i j}(t) z_{j}+f_{i}\left(z_{1}, \cdots, z_{N}, d z_{1} / d t, \cdots, d z_{N} / d t, t\right), & \\
d y_{i} / d t=\sum_{j=1}^{N} a_{i j}(t) y_{j}, & i=1,2, \cdots, N, \\
& i=1,2, \cdots, N .
\end{array}
$$


Comparing the two systems, two viewpoints are possible. One may regard (2.2) as the original equation, and (2.1) as a perturbed equation, or one may reverse roles, and regard (2.2) as an approximate equation, and (2.1) as the original. Mathematically, there is no distinction, as almost always the properties of the simpler equation will be investigated first.

The latter point of view originated with Poincare in a series of papers on curves satisfying differential equations. The former point of view, for the case where the $f_{i}$ are linear forms in the $z_{k}$, is also intimately connected with pioneer work of Poincaré, and has a long history behind it. This will not be discussed here. We mention Perron [10], Hukuwara [6], and Cesari [3], for results and references.

Notation. Statements and proofs are simplified considerably if vectormatrix notation is used in dealing with systems of differential equations.

Capital letters, $A, B, \cdots, X, Y$, will denote matrices; small letters without subscript will denote column vectors. Positive constants whose exact values are not necessary for the proof will be denoted by $c_{1}, c_{2}$, and so on. The components of $y$ will be denoted by $y_{k}$, and the elements of $A$ by $a_{i j}$. The norm of a vector and a matrix are defined as follows:

$$
\|y\|=\sum_{k=1}^{N}\left|y_{k}\right|, \quad\|A\|=\sum_{i, j=1}^{N}\left|a_{i j}\right| \text {. }
$$

It is easily verified that

$$
\begin{aligned}
\|y+z\| & \leqq\|y\|+\|z\|, & \|A+B\| & \leqq\|A\|+\|B\|, \\
\|A y\| & \leqq\|A\|\|y\|, & \|A B\| & \leqq\|A\|\|B\| .
\end{aligned}
$$

A vector function, $y(t)$, or a matrix function, $Y(t)$, is said to be bounded if its norm is bounded as $t \rightarrow \infty$. Similarly it is said to tend to zero as $t$ if its norm tends to zero. The terms continuous, equi-continuous, and so forth can be defined in terms of the norm, and the definitions are as for ordinary functions of one variable.

The system (2.1) can now be written

$$
d z / d t=A z+f(z, d z / d t, t),
$$

where $z$ is the column vector whose components are $z_{k}, A$ is the matrix $\left(a_{i j}\right)$, and $f(z, d z / d t, t)$ represents the column vector whose components are the $f_{i}$.

Usually the elements of $A$ will be constants. If not, they will be assumed to be well-behaved functions, continuous over any finite interval. Weaker conditions could be imposed, but as we are principally interested in the behavior of the solutions at infinity, there seems to be no point in discussing finite singularities.

When $A$ is constant, the behavior of the solutions of

$$
d y / d t=A y
$$


at infinity is completely determined by the nature of the characteristic roots of $A$. The principal subject of the first part of the paper will be the influence of $A, f(z, d z / d t, t)$, and the initial conditions upon the behavior at infinity of the solutions of (2.5).

The vector equation, (2.6), has $N$ linearly independent solutions, $y^{(1)}, y^{(2)}, \cdots, y^{(N)}$. These $N$ linearly independent solutions can be chosen so that the $N \times N$ matrix, $Y$, whose columns are the $y^{(k)}$, satisfies the initial condition, $Y(0)=I$. It is clear then that $Y$ satisfies

$$
d Y / d t=A Y, \quad Y(0)=I .
$$

Any other nonsingular solution of (2.7) is of the form $Y C$, where $C$ is a nonsingular constant matrix.

The following uniqueness result will be required occasionally. We state it as:

LEMMA 1. If each component of $A z+f(z, t)$ is continuous, and satisfies a Lipschitz condition for the $z_{k}$ lying in a finite domain, $D$, of the $\left(z_{k}, t\right)$ space, and if $\left(z_{0}, t_{0}\right)$ is in $D$, and $z_{1}(t), z_{2}(t)$ are two solutions of $(2.5)$ in an interval about $t_{0}$ such that $z_{0}=z_{1}\left(t_{0}\right)=z_{2}\left(t_{0}\right)$, then $z_{1}(t) \equiv z_{2}(t)$.

A fundamental characteristic of this paper is the conversion of the differential equation into an integral equation. This device, introduced by Liouville, brought into prominence this important type of integral equation. The procedure for converting the differential equation into the required form is discussed in the following lemma.

LEMMA 2. The solution of

$$
d z / d t=A z+w(t), \quad z\left(t_{0}\right)=y\left(t_{0}\right),
$$

where $y(t)$ is a solution of $d y / d t=A y$, is

$$
z=y+\int_{t_{0}}^{t} Y(t) Y^{-1}\left(t_{1}\right) w\left(t_{1}\right) d t_{1} .
$$

If $A$ is a constant matrix, this reduces to

$$
z=y+\int_{t_{0}}^{t} Y\left(t-t_{1}\right) w\left(t_{1}\right) d t_{1} .
$$

In the applications that will be made of this lemma, $w$ will contain the unknown function, $z$, and thus (2.9) will be an integral equation.

Proof. Although the result is well known, the proof will be given for the sake of completeness. It is obtained by the method of variation of parameters. Let $z=Y u$. Then

$$
Y^{\prime} u+Y u^{\prime}=A Y u+w
$$


or

$$
u^{\prime}=Y^{-1} w, \quad u=v+\int_{t_{0}}^{t} Y^{-1} w d t_{1},
$$

where $v$ is the first column of the identical matrix, $I$. Thus

$$
z=y+\int_{t_{0}}^{t} Y(t) Y^{-1}\left(t_{1}\right) w\left(t_{1}\right) d t_{1} .
$$

If $A$ is constant, $Y\left(t-t_{1}\right)=Y(t) Y^{-1}\left(t_{1}\right)$. For when $A$ is constant, $Y\left(t-t_{1}\right)$ is a solution of (2.7) equal to $I$ at $t=t_{1}$, and simultaneously $Y(t) Y^{-1}\left(t_{1}\right)$ is a solution of (2.7) satisfying the same boundary condition. Hence both coincide, column by column.

3. The characteristic roots of $A$ all have negative real parts. The main result of this section is contained in the following theorem.

THEOREM 2. Consider the differential equation

$$
d z / d t=(A+B) z+B_{1} d z / d t+f(z, d z / d t, t),
$$

where

(3.2) (1) The characteristic roots of $A$ have all negative real parts,

(2) $\|B\| \leqq c_{1},\left\|B_{1}\right\| \leqq c_{1}, c_{1}$ depending upon $A$,

(3) $\mid \partial f(z, w, t) / \partial z_{k} \|=o(1)$ as $\|z\|+\|w\| \rightarrow 0$, uniformly in $t$,

(4) $\left\|\partial f(z, w, t) / \partial w_{k}\right\|=o(1)$ as $\|z\|+\|w\| \rightarrow 0$, uniformly in $t$.

Under these conditions, there exists an $N$-dimensional manifold of solutions of (3.1) which approaches 0 as $t \rightarrow \infty$.

If the right-hand side of (3.1) does not contain dz/dt, every solution of (3.1) whose initial values are close enough to the zero solution is uniformly bounded for all $t \geqq 0$, and approaches 0 as $t \rightarrow \infty$.

Proof. Let $y$ be the solution of

$$
d y / d t=A y, \quad y(0)=y_{0},
$$

where the order of magnitude of $\left\|y_{0}\right\|$ will be prescribed below. We shall choose $z$ to have the initial value $y_{0}$. We first show that $\|y(t)\|<c_{3}\left\|y_{0}\right\|, t \geqq t_{0} \geqq 0$, where $c_{3}$ is a constant depending only upon $A$. The solution of (3.3) can be expressed as $y=Y(t) y_{0}$. Thus $\|y\| \leqq\|Y(t)\|\left\|y_{0}\right\| \leqq c_{3}\left\|y_{0}\right\|$, since in consequence of (3.2) (1), $\|Y(t)\|$ is bounded.

Using Lemma 2, we now convert (3.1) into the integral equation

$$
\begin{aligned}
z=y & +\int_{0}^{t} Y\left(t-t_{1}\right) B\left(t_{1}\right) z\left(t_{1}\right) d t_{1}+\int_{0}^{t} Y\left(t-t_{1}\right) B_{1}\left(t_{1}\right) \frac{d z\left(t_{1}\right)}{d t} d t_{1} \\
& +\int_{0}^{t} Y\left(t-t_{1}\right) f\left(z, d z / d t_{1}, t_{1}\right) d t_{1} .
\end{aligned}
$$


A solution of this integral equation will be a solution of (3.1) with the initial value $z(0)=y(0)=y_{0}$. If we show that a bounded solution of (3.4) exists, under the assumptions of (3.2) and with $\|z(0)\|$ small enough, we shall have demonstrated the existence of the required $N$-dimensional manifold, since we can choose $y=\sum_{k=1}^{N} a_{k} y^{(k)}$ where the $y^{(k)}$ are $N$ linearly independent solutions of $d y / d t=A y$, and $\sum_{k=1}^{N}\left|a_{k}\right|$ is sufficiently small. The $N$ constants $a_{k}, k=1,2, \cdots, N$, generate the manifold of solutions.

We now use the method of successive approximations to obtain a solution of (3.4). Define a sequence of vector functions as follows:

$z_{0}=y$,

$$
\begin{aligned}
& z_{n+1}=y+\int_{0}^{t} Y\left(t-t_{1}\right) B\left(t_{1}\right) z_{n} d t_{1}+\int_{0}^{t} Y\left(t-t_{1}\right) B_{1}\left(t_{1}\right) \frac{d z_{n}}{d t} d t_{1} \\
& +\int_{0}^{t} Y\left(t-t_{1}\right) f\left(z_{n}, d z_{n} / d t_{1}, t_{1}\right) d t_{1} \\
& n \geqq 0 \text {. }
\end{aligned}
$$

The conditions imposed upon $A$ show that not only does $\|Y\| \rightarrow 0$ as $t \rightarrow \infty$, but also $\int_{0}^{\infty}\|Y(u)\| d u<\infty$. This becomes clear when we realize that each element of $Y$ is a sum of terms of the form $e^{\lambda t}(P(t) \cos \mu t+Q(t) \sin \mu t)$ where $P(t)$ and $Q(t)$ are polynomials of degree at most $N-1$, and $\lambda, \mu$ correspond to the characteristic root $\lambda+i \mu$, with $\lambda<0$.

The first step in the proof will be to show that the $z_{n}$ and $d z_{n} / d t$ are uniformly bounded, provided that $\|z(0)\|$ is sufficiently small. Set $c_{4}=c_{3}\left\|y_{0}\right\|$. We have $\left\|z_{0}\right\|=\|y\| \leqq c_{3}\left\|y_{0}\right\|=c_{4}$, and $\left\|d z_{0} / d t\right\|=\left\|A z_{0}\right\| \leqq\|A\|\left\|z_{0}\right\|<\|A\| c_{4}$. Let us now assume that $\left\|z_{k}\right\| \leqq 2 c_{4}, k=0,1, \cdots, n$, and show that this also holds for $n+1$; similarly we assume that $\left\|d z_{k} / d t\right\| \leqq 3\|A\| c_{4}, k=0,1, \cdots, n$, and show that this holds for $n+1$. We have

$$
\begin{aligned}
\left\|z_{n+1}\right\| \leqq\|y\| & +\int_{0}^{t}\left\|Y\left(t-t_{1}\right)\right\|\left\|B\left(t_{1}\right)\right\|\left\|z_{n}\right\| d t_{1} \\
& +\int_{0}^{t}\left\|Y\left(t-t_{1}\right)\right\|\left\|B_{1}\left(t_{1}\right)\right\|\left\|d z_{n} / d t_{1}\right\| d t_{1} \\
& +\int_{0}^{t}\left\|Y\left(t-t_{1}\right)\right\|\left\|f\left(z_{n}, d z_{n} / d t_{1}, t_{1}\right)\right\| d t_{1} .
\end{aligned}
$$

In consequence of conditions (3) and (4) of (3.2), we have

$$
\left\|f\left(z_{n}, \frac{d z_{n}}{d t}, t\right)\right\| \leqq c_{6}\left(\left\|z_{n}\right\|+\left\|\frac{d z_{n}}{d t}\right\|\right) \leqq\left(2 c_{4}+3\|A\| c_{4}\right) c_{6}
$$

where $c_{B}$ is a constant that can be made as small as desired by starting with $\left\|z_{n}\right\|+\left\|d z_{n} / d t\right\|$ sufficiently small. Thus, from (3.6), there results 


$$
\begin{aligned}
&\left\|z_{n+1}\right\| \leqq c_{4}+\int_{0}^{t}\left\|Y\left(t-t_{1}\right)\right\|\left\|B\left(t_{1}\right)\right\|\left(2 c_{4}\right) d t_{1} \\
&+\int_{0}^{t}\left\|Y\left(t-t_{1}\right)\right\|\left\|B_{1}\left(t_{1}\right)\right\|\left(2 c_{4}\right) d t_{1} \\
&+\int_{0}^{t}\left\|Y\left(t-t_{1}\right)\right\|\left(2 c_{4}+3\|A\| c_{4}\right) c_{8} d t_{1} \\
& \leqq+2 c_{4}\left[2 c_{1} \int_{0}^{\infty}\|Y(u)\| d u\right]+2 c_{4}(1+3\|A\|) \int_{0}^{\infty}\|Y(u)\| d u \\
& \leqq 2 c_{4}
\end{aligned}
$$

provided that $c_{1}$ and $c_{8}$ are sufficiently small. To obtain the bound for $d z_{n} / d t$, we cbserve that the recurrence relation (3.5) is equivalent to the recurrence relation

$$
d z_{n+1} / d t=A z_{n+1}+B z_{n}+B_{1} d z_{n} / d t+f\left(z_{n}, d z_{n} / d t, t\right)
$$

Thus

$$
\begin{aligned}
\left\|d z_{n+1} / d t\right\| \leqq & \|A\|\left\|z_{n+1}\right\|+\|B\|\left\|z_{n}\right\|+\left\|B_{1}\right\|\left\|d z_{n} / d t\right\| \\
& +\left\|f\left(z_{n}, d z_{n} / d t, t\right)\right\| \\
\leqq & 2\|A\| c_{4}+2 c_{1} c_{4}+c_{1}\left(3\|A\| c_{4}\right)+\left(2 c_{4}+3\|A\| c_{4}\right) c_{8} \\
\leqq & 3\|A\| c_{4} .
\end{aligned}
$$

We can fix the sizes of $\|B\|$ and $\left\|B_{1}\right\|$, and thus of $c_{1}$, and we can choose the magnitude of the initial value, $\left\|y_{0}\right\|$, so as to make $c_{1}$ the right order of magnitude. Thus we have shown that the bounds are uniform in $n$ and $z$.

The next step is the standard one, namely to prove the convergence of $\sum_{n=0}^{\infty}\left(z_{n+1}-z_{n}\right)$. We shall consider the majorizing series $\sum_{n=0}^{\infty}\left(\left\|z_{n+1}-z_{n}\right\|\right.$ $\left.+\left\|d z_{n+1} / d t-d z_{n} / d t\right\|\right)$. We have

$$
\begin{aligned}
\left\|z_{n+1}-z_{n}\right\|= & \|\left[\int_{0}^{t} Y\left(t-t_{1}\right) B\left(t_{1}\right)\left(z_{n}-z_{n-1}\right) d t_{1}\right. \\
& +\int_{0}^{t} Y\left(t-t_{1}\right) B_{1}\left(t_{1}\right)\left(z_{n}-z_{n-1}\right) d t_{1} \\
& +\int_{0}^{t} Y\left(t-t_{1}\right)\left(f\left(z_{n}, d z_{n} / d t_{1}, t_{1}\right)\right. \\
& \left.\left.-f\left(z_{n-1}, d z_{n-1} / d t_{1}, t_{1}\right)\right) d t_{1}\right] \| .
\end{aligned}
$$

Thus 


$$
\begin{aligned}
\left\|z_{n+1}-z_{n}\right\| \leqq & \int_{0}^{t}\left\|Y\left(t-t_{1}\right)\right\|\left\|B\left(t_{1}\right)\right\|\left\|z_{n}-z_{n-1}\right\| d t_{1} \\
& +\int_{0}^{t}\left\|Y\left(t-t_{1}\right)\right\|\left\|B_{1}\left(t_{1}\right)\right\|\left\|z_{n}-z_{n-1}\right\| d t_{1} \\
& +c_{7} \int_{0}^{t}\left\|Y\left(t-t_{1}\right)\right\|\left(\left\|z_{n}-z_{n-1}\right\|+\left\|\frac{d z_{n}}{d t}-\frac{d z_{n-1}}{d t}\right\|\right) d t_{1} .
\end{aligned}
$$

From conditions (3), (4) of (3.2) it is seen that $c_{7}$ can be made as small as desired by choosing the bounds on $z_{n}$ and $d z_{n} / d t$ small enough. These bounds in turn depend upon $z(0)=y(0)$, and thus are at our disposal. We also have

$$
\begin{aligned}
d z_{n+1} / d t-d z_{n} / d t=A\left(z_{n+1}-z_{n}\right)+f\left(z_{n}, d z_{n} / d t, t\right) & \\
& -f\left(z_{n-1}, d z_{n-1} / d t, t\right),
\end{aligned}
$$

whence

$$
\begin{aligned}
& \left\|d z_{n+1} / d t-d z_{n} / d t\right\| \leqq\|A\|\left\|z_{n+1}-z_{n}\right\| \\
& +c_{8}\left(\left\|z_{n}-z_{n-1}\right\|+\left\|d z_{n} / d t-d z_{n-1} / d t\right\|\right) \text {. }
\end{aligned}
$$

From (3.11) we obtain

$$
\begin{aligned}
\left\|z_{n+1}-z_{n}\right\| \leqq & \max _{0 \leqq t_{1} \leqq}\left\|z_{n}-z_{n-1}\right\|\left[2 c_{1} \int_{0}^{\infty}\|Y(u)\| d u+c_{7} \int_{0}^{\infty}\|Y(u)\| d u\right] \\
& +\max _{0 \leqq i_{1} \leqq t}\left\|d z_{n} / d t-d z_{n-1} / d t\right\|\left[c_{7} \int_{0}^{\infty}\|Y(u)\| d u\right] .
\end{aligned}
$$

Using this estimate and (3.13) we obtain

$$
\begin{aligned}
& \left\|\frac{d z_{n+1}}{d t}-\frac{d z_{n}}{d t}\right\| \\
& \leqq \\
& \quad\left[c_{8}+\left(2 c_{1}+c_{7}\right)\|A\| \int_{0}^{\infty}\|Y(u)\| d u \max _{0 \leqq t_{1} \leq t}\left\|z_{n}-z_{n-1}\right\|\right. \\
& \quad+\left[c_{8}+c_{7}\|A\| \int_{0}^{\infty}\|Y(u)\| d u\right] \max _{0 \leq t_{1} \leq t}\left\|\frac{d z_{n}}{d t}-\frac{d z_{n-1}}{d t}\right\| .
\end{aligned}
$$

Adding (3.16) and (3.15), we have the desired result

$$
\begin{aligned}
\max _{0 \leqq t_{1} \leq t}\left[\left\|z_{n+1}-z_{n}\right\|+\| \frac{d z_{n+1}}{d t}\right. & \left.-\frac{d z_{n}}{d t} \|\right] \\
& \leqq c_{9} \max _{0 \leqq t_{1} \leq t}\left[\left\|z_{n}-z_{n-1}\right\|+\left\|\frac{d z_{n}}{d t}-\frac{d z_{n-1}}{d t}\right\|\right]
\end{aligned}
$$


where $c_{9}$ will be less than 1 , if $c_{1}, c_{7}$, and $c_{8}$ are small enough. Thus the majorizing series $\sum_{n=0}^{\infty}\left[\left\|z_{n+1}-z_{n}\right\|+\left\|d z_{n+1} / d t-d z_{n} / d t\right\|\right]$ converges, since its terms will be majorized by the terms of the geometric series $\left\|y_{0}\right\| \sum_{n=0}^{\infty} c_{9}^{n}$, and the series $\sum_{n=0}^{\infty}\left(z_{n+1}-z_{n}\right)$ converges uniformly to $z-y$, where $z$ will be a solution of the differential equation (3.1).

To show that $\|z\| \rightarrow 0$ as $t \rightarrow \infty$, we show that $\|z\| \leqq c_{10} e^{-\lambda_{1} t}$, where $-\lambda_{1}$ is negative, and greater than the maximum of the real parts of the characteristic roots of $A$, and $c_{10}$ is a constant depending only upon $\lambda_{1}$. This is proved by induction for each $z_{n}$, it being clearly true for $z_{0}$, and the proof is similar to the above.

If, as in the below-mentioned case, $d z / d t$ is lacking on the right-hand side of the equation (3.1), $a$ solution obtained by the method of successive approximations is the solution of the differential equation, and thus the conclusion is, as in Perron's more inclusive theorem, that every solution whose initial values are close enough to the zero solution remains uniformly bounded and approaches 0 as $t \rightarrow \infty$.

As cited in the introduction, Levinson, in a letter to the author, has shown how to derive the boundedness of any solution of (3.1) from the integral equation (3.4), using only the condition that $f(z, w, t)=o(\|z\|+\|w\|)$ as $\|z\|+\|w\| \rightarrow 0$.

The result for $B=B_{1}=0$, and all the components of $f(z, d z / d t, t)$ power series in the $z_{k}$ only, beginning with second degree terms with constant coefficients, is due to Poincaré [18] for $N=2$, and to Liapounoff for general $N \geqq 1$. It is easy to see that all the conditions of (3.2) are satisfied under these conditions.

As the following example shows, even in the simplest cases, a boundedness condition on the initial values is necessary for the truth of the theorem. Consider the equation

$$
d y / d t=-y+y^{2}, \quad y(0)=2,
$$

letting $y$ for the moment be an ordinary function of one variable, $t$. The solution of (3.18) is given by $y=2 /\left(2-e^{t}\right)$, which approaches $\infty$ as $t \rightarrow \log 2$.

We now wish to consider a generalization, considered previously for their types of equations by Liapounoff and Perron. We assume that $k, k \leqq N$, of the characteristic roots have negative real parts. We can then state:

THEOREM 3. Consider the differential equation (3.1) with conditions (2), (3), (4) of (3.2) satisfied, and let $k, k \leqq N$, of the characteristic roots of $A$ have negative real parts. Then there exists a $k$-dimensional manifold of solutions of (3.1) which approach 0 as $t \rightarrow \infty$.

Proof. As in the previous proof we begin with the recurrence relation (3.5). However, since only $k$ of the characteristic roots have negative real parts, it will be necessary to modify this slightly to eliminate those solutions of 
$d y / d t=A y$ which do not approach 0 as $t \rightarrow \infty$. The first step is to decompose $Y$ into the sum of two other matrices. To this end we consider the form of any element, $y_{i j}$, of $Y . y_{i j}$ is the sum of terms of the form $e^{\lambda t}(P(t) \cos \mu t+Q(t)$ $\cdot \sin \mu t)$ w here $\lambda, \mu$ correspond to the characteristic root $\lambda+i \mu$, and $P(t), Q(t)$ are polynomials of degree at most $N-1$. Let the sum of those terms where $\lambda<0$ be denoted by $u_{i j}$, and the sum of those terms remaining where $\lambda \geqq 0$ be denoted by $v_{i j}$. Let $Y_{1}=\left(u_{i j}\right), Y_{2}=\left(v_{i j}\right)$. Then $Y=Y_{1}+Y_{2}$. Since there can be no cancellation between terms with $\lambda<0$ and terms with $\lambda \geqq 0$, we must have

$$
d Y_{1} / d t=A Y_{1}, \quad d Y_{2} / d t=A Y_{2} .
$$

Now choose $k$ linearly independent solutions, $w^{(1)}, w^{(2)}, \cdots, w^{(k)}$, of $d y / d t=A y$, whose norms approach zero as $t \rightarrow \infty$. If $y=\sum_{r=1}^{k} a_{r} w^{(r)},\|y\|$ can be made as small as desired by taking $\sum_{n=1}^{k}\left|a_{n}\right|$ sufficiently small. The $k$-dimensional manifold will be generated by the $k$ constants $a_{r}$. Let $\max t \geq 0\|y\|=c_{3}$, where the order of magnitude will be specified below.

We now modify the recurrence relation of (3.5) as follows:

$$
\begin{aligned}
z_{n+1}=y & +\int_{0}^{t} Y\left(t-t_{1}\right) B\left(t_{1}\right) z_{n} d t_{1}-\int_{0}^{\infty} Y_{2}\left(t-t_{1}\right) B\left(t_{1}\right) z_{n} d t_{1} \\
& +\int_{0}^{t} Y\left(t-t_{1}\right) B_{1}\left(t_{1}\right) \frac{d z_{n}}{d t_{1}} d t_{1}-\int_{0}^{\infty} Y_{2}\left(t-t_{1}\right) B_{1}\left(t_{1}\right) \frac{d z_{n}}{d t_{1}} d t_{1} \\
& +\int_{0}^{t} Y\left(t-t_{1}\right) f\left(z_{n}, \frac{d z_{n}}{d t_{1}}, t_{1}\right) d t_{1} \\
& -\int_{0}^{\infty} Y_{2}\left(t-t_{1}\right) f\left(z_{n}, \frac{d z_{n}}{d t_{1}}, t_{1}\right) d t_{1} .
\end{aligned}
$$

Since $Y_{2}\left(t-t_{1}\right) f\left(z\left(t_{1}\right), d z / d t_{1}, t_{1}\right)$ is a solution of $d y / d t=A y$ for any $t_{1}$, $\int_{0}^{\infty} Y\left(t-t_{1}\right) f\left(z, d z / d t_{1}, t_{1}\right) d t_{1}$ is also a solution, and therefore if the sequence $z_{n}$. converges, it converges to a solution of (3.1).

Using the relation $Y=Y_{1}+Y_{2}$, the recurrence relation becomes

$$
\begin{aligned}
z_{0}=y & \\
z_{n+1}=y & +\int_{0}^{t} Y_{1}\left(t-t_{1}\right) B\left(t_{1}\right) z_{n} d t_{1}-\int_{t}^{\infty} Y_{2}\left(t-t_{1}\right) B\left(t_{1}\right) z_{n} d t_{1} \\
& +\int_{0}^{t} Y_{1}\left(t-t_{1}\right) B_{1}\left(t_{1}\right) \frac{d z_{n}}{d t_{1}}-\int_{t}^{\infty} Y_{2}\left(t-t_{1}\right) B_{1}\left(t_{1}\right) \frac{d z_{n}}{d t_{1}} d t_{1} \\
& +\int_{0}^{t} Y_{1}\left(t-t_{1}\right) f\left(z_{n}, \frac{d z_{n}}{d t_{1}}, t_{1}\right) d t_{1} \\
& -\int_{t}^{\infty} Y_{2}\left(t-t_{1}\right) f\left(z_{n}, \frac{d z_{n}}{d t_{1}}, t_{1}\right) d t_{1}, \quad n \geqq 0 .
\end{aligned}
$$


The first step, again, is to show that the sequences $z_{n}, d z_{n} / d t$ are uniformly bounded. Instead of a constant bound, we obtain an exponential bound involving $t$, which serves to prove the statement concerning the approach to the zero solution as $t \rightarrow \infty$. Let $-\lambda$ be the least negative of the negative real parts of the characteristic roots of $A$. Choose, $\lambda_{1}, \lambda_{2}$ so that

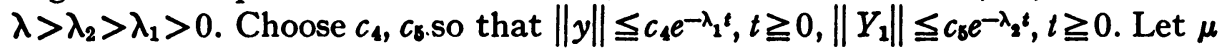
be the maximum of the real parts of all the characteristic roots of $A$, and choose $\mu_{1}>\mu \geqq 0$. Choose $c_{6}$ to satisfy $\left\|Y_{2}\right\| \leqq c_{8} e^{\mu t}, t \geqq 0$.

We now assume that $\left\|z_{k}\right\| \leqq 2 c_{4} e^{-\lambda_{11} t},\left\|d z_{k} / d t\right\| \leqq 3 c_{A}\|A\| e^{-\lambda_{1} t}$, for $k=0,1$, $\cdots, n$. The inequality is certainly true when $k=0$, and we now show that it holds for $n+1$ as well. We have

$$
\begin{aligned}
\left\|z_{n+1}\right\| \leqq\|y\| & +\int_{0}^{t}\left\|Y_{1}\left(t-t_{1}\right)\right\|\left\|B\left(t_{1}\right)\right\|\left\|z_{n}\right\| d t_{1} \\
& +\int_{t}^{\infty}\left\|Y_{2}\left(t-t_{1}\right)\right\|\left\|B_{1}\left(t_{1}\right)\right\|\left\|z_{n}\right\| d t_{1} \\
& +\int_{0}^{t}\left\|Y_{1}\left(t-t_{1}\right)\right\|\left\|B_{1}\left(t_{1}\right)\right\|\left\|\frac{d z_{n}}{d t_{1}}\right\| d t_{1} \\
& +\int_{t}^{\infty}\left\|Y_{2}\left(t-t_{1}\right)\right\|\left\|B_{1}\left(t_{1}\right)\right\|\left\|\frac{d z_{n}}{d t_{1}}\right\| d t_{1} \\
& +\int_{0}^{t}\left\|Y_{1}\left(t-t_{1}\right)\right\|\left\|f\left(z_{n}, \frac{d z_{n}}{d t_{1}}, t_{1}\right)\right\| \\
& +\int_{t}^{\infty}\left\|Y_{2}\left(t-t_{1}\right)\right\|\left\|f\left(z_{n}, \frac{d z_{n}}{d t_{1}}, t_{1}\right)\right\| d t_{1} \\
\leqq & c_{4} e^{-\lambda_{1} t}+2 c_{1} c_{4} c_{5} \int_{0}^{t} e^{-\lambda_{2}\left(t-t_{1}\right)-\lambda_{1} t_{1}} d t_{1}+2 c_{1} c_{4} c_{6} \int_{t}^{\infty} e^{\mu_{1}\left(t-t_{1}\right)-\lambda_{1} t_{1}} d t_{1} \\
& +3 c_{1} c_{4} c_{5}\|A\| \int_{0}^{t} e^{-\lambda_{2}\left(t-t_{1}\right)-\lambda_{1} t_{1}} d t_{1}+3 c_{1} c_{4} c_{6}\|A\| \int_{t}^{\infty} e^{e_{1}\left(t-t_{1}\right)-\lambda_{1} t_{1}} d t_{1} \\
& +c_{1}\left(2 c_{4}+3 c_{4}\|A\|\right) c_{5} c_{7} \int_{0}^{t} e^{-\lambda_{2}\left(t-t_{1}\right)-\lambda_{1} t_{1}} d t_{1} \\
\leqq & +c_{1}\left(2 c_{4}+3 c_{4}\|A\|\right) c_{8} c_{7} \int_{t}^{\infty} e^{\mu_{1}\left(t-t_{1}\right)-\lambda_{1} t_{1}} d t_{1} \\
&
\end{aligned}
$$

provided that $c_{1}$ and $c_{7}$ are small enough. Also, since

$$
d z_{n+1} / d t=A z_{n+1}+B z_{n}+B_{1} d z_{n} / d t+f\left(z_{n}, d z_{n} / d t, t\right)
$$


we have

$$
\begin{aligned}
\left\|\frac{d z_{n+1}}{d t}\right\| \leqq & \|A\|\left\|z_{n}\right\|+\|B\|\left\|z_{n}\right\|+\left\|B_{1}\right\|\left\|\frac{d z_{n}}{d t}\right\|+\left\|f\left(s_{n}, \frac{d z_{n}}{d t}, t\right)\right\| \\
\leqq & 2\|A\| c_{4} e^{-\lambda_{1} t}+2 c_{1} c_{4} e^{-\lambda_{1} t}+3 c_{1} c_{4}\|A\| e^{-\lambda_{1} t} \\
& +c_{7}\left(2 c_{4}+3\|A\| c_{4}\right) e^{-\lambda_{1} t} \\
\leqq & 3\|A\| c_{4} e^{-\lambda_{1} t}
\end{aligned}
$$

again if $c_{1}, c_{7}$ are small enough.

Thus we have obtained uniform bounds for $z_{n}$ and $d z_{n} / d t$. The next step is to show the convergence of the series $\sum_{n=0}^{\infty}\left(z_{n+1}-z_{n}\right)$, and as there is no novelty to this we omit it.

The condition that $\|f(z, w, t)\|=o(\|z\|+\|w\|)$, uniformly in $t$, is far too restrictive. Actually, it suffices that $f(z, w, t)$ be majorized by $g\left(z e^{\lambda t}, w e^{\lambda t}, t\right)$, where $\|g(z, w, t)\|=o(\|z\|+\|w\|)$ as $\|z\|+\|w\| \rightarrow 0$, where $\lambda$ is less than the minimum of the absolute values of the real parts of the characteristic roots of $A$, assuming, of course, that all the real parts are negative. If this condition is satisfied, a change of variable, $z e^{\lambda t}=z^{\prime}$, will bring the problem into the form treated in Theorem 2 .

4. The characteristic roots of $A$ all have nonpositive real parts. Allowing the characteristic roots of $A$ to have zero real parts makes it necessary to impose more stringent restrictions upon $B, B_{1}$, and $f(z, d z / d t, t)$. It is no longer true that $\|Y\|$ is integrable over $(0, \infty)$. To compensate for this, we shall consider only matrices $B$ and $B_{1}$ which have norms integrable over $(0, \infty)$, and functions $f(z, d z / d t, t)$ which are also majorized by functions integrable over this range. These conditions are rather harsh, but it is easy to construct examples which show that they cannot be bettered in general. We refer to Cesari [3] and Wintner [16], for discussion and examples of this topic. Liapounoff [8] considered the case where two characteristic roots are pure complex and, by a change of variable, reduces it to the case of one zero root of the characteristic equation. This method is due to Poincare. We shall not discuss these special cases, important as they are, but shall attempt to derive general criteria.

One consequence of these heavier restrictions is that the stability properties of the solutions vary with the initial value, $t_{0}$. Let us call a neighborhood of the origin in the $\left(y_{1}, y_{2}, \cdots, y_{n}\right)$-plane the set of all points $\left(y_{1}, y_{2}, \cdots, y_{N}\right)$ which satisfy an inequality $\sum_{k=1}^{N}\left|y_{k}\right| \leqq c$. For the case where all the characteristic roots had negative real parts there was a certain neighborhood of the origin in the $z_{k}$-plane with the property that if at any time, $t_{0}$, the solution was inside this neighborhood, it approached the zero solution, or origin, as $t \rightarrow \infty$. This certain neighborhood was independent of $t_{0}$. We shall see that in the present case the situation is quite different. Let us call a neighborhood of the origin a stability neighborhood, designated by $S\left(t_{0}\right)$, if any solution 
which lies inside it for $t=t_{0}$ remains inside some fixed neighborhood, independent of $t_{0}$, for $t \geqq t_{0}$. Let the $c$ of the inequality $\sum_{k=1}^{N}\left|y_{k}\left(t_{0}\right)\right| \leqq c$ be the "radius" of this neighborhood, and call it $R\left(t_{0}\right)$.

The previous case led to the fact that $R\left(t_{0}\right) \geqq c_{1}$, where $c_{1}$ was independent of $t_{0}$. In this section we shall show that under certain conditions of the type discussed above imposed upon $B, B_{1}$, and $f(z, d z / d t, t), R\left(t_{0}\right) \rightarrow \infty$ as $t_{0} \rightarrow \infty$. We shall further show that the manifold of bounded solutions can be split into two sub-manifolds; one manifold consisting of those solutions which tend to zero as $t \rightarrow+\infty$, and one manifold consisting of solutions which tend to almost-periodic functions of $t$ as $t \rightarrow+\infty$. The result to be proved is:

THEOREM 4. Consider the differential equation of (3.1) where:

(4.1) (1) $A$ is a constant matrix, and all solutions of $d y / d t=A y$ are bounded.

(2) $\left\|f_{z_{k}}(z, \cdot w, t)\right\|+\left\|f_{w_{k}}(z, w, t)\right\| \leqq h(t) g(c)$, for $\|z\|+\|z o\| \leqq c, 1 \leqq k \leqq N$.

(3) $\int_{0}^{\infty} h(t) d t<\infty, g(c) \rightarrow 0$, as $c \rightarrow 0$.

(4) $\int_{0}^{\infty}\|B\| d t \leqq c_{1}, \int_{0}^{\infty}\left\|B_{1}\right\| d t \leqq c_{1}$, where $c_{1}$ will depend upon $A$ and $f(z, w, t)$.

Under these conditions there exists an $N$-dimensional manifold of bounded solutions of (3.1).

If the right-hand side does not contain $d z / d t$, every solution, $z$, of (3.1) for which $\|z(0)\|$ is small enough is uniformly bounded for all $t \geqq 0$. If $A$ has $k$ roots, $k \leqq N$, whose real parts are negative, there is a $k$-dimensional manifold of solutions which approaches 0 as $t \rightarrow \infty$, and an $(N-k)$-dimensional manifold of solutions which approach almost-periodic functions as $t \rightarrow+\infty$.

It is only necessary that condition (2) of (4.1) be satisfied for small $c$.

Proof. We begin with the proof of the stability property. The same recurrence relation that appeared in the proof of Theorem 2 is used here. Let us consider the initial value of $t$ to be $t_{0}$ instead of 0 . We have to prove the uniform convergence of the sequence of vector functions given by

$$
\begin{aligned}
z_{0}=y, & \\
z_{n+1}=y & +\int_{t_{0}}^{t} Y\left(t-t_{1}\right) B\left(t_{1}\right) z_{n} d t_{1}+\int_{t_{0}}^{t} Y\left(t-t_{1}\right) B_{1}\left(t_{1}\right) \frac{d z_{n}}{d t_{1}} d t_{1} \\
& +\int_{t_{0}}^{t} Y\left(t-t_{1}\right) f\left(z_{n}, d z_{n} / d t_{1}, t_{1}\right) d t_{1}, \quad n \geqq 0 .
\end{aligned}
$$

The proof now proceeds as before with the integrability of $\|B(t)\|,\left\|B_{1}(t)\right\|$, and $h(t)$ replacing the integrability of $\|Y(t)\|$. Here the only property required of $\|Y(t)\|$ is that it be bounded for all $t \geqq 0$. This is a consequence of (4.1) (1) which implies that all the characteristic roots of $A$ have nonpositive real parts, and that multiple characteristic roots which have zero real parts can only occur in such a way as to leave $\|Y(t)\|$ still uniformly bounded for $t \geqq 0$. An equivalent way of stating this is to say that those elementary 
divisors corresponding to characteristic roots having zero real parts are simple.

Since $\int_{0}^{\infty}\|B(t)\| d t, \int_{0}^{\infty}\left\|B_{1}(t)\right\| d t$, and $\int_{0}^{\infty} h(t) d t$ all converge, we have each of the functions $\int_{t_{1}}^{\infty}\|B(t)\| d t, \int_{t_{1}}^{\infty}\left\|B_{1}(t)\right\| d t, \int_{t_{1}}^{\infty} h(t) d t \rightarrow 0$ as $t_{1} \rightarrow \infty$. From the way these quantities enter into the proof, it is seen that we may allow $\left\|z\left(t_{0}\right)\right\|$ to be larger and larger for increasing $t_{0}$, depending upon the rate of decrease of the three integrals. The sequence of (4.2) will converge to a bounded solution as before. We see then that the stability neighborhood becomes larger as $t_{0}$ becomes larger, and approaches $\infty$ as $t_{0} \rightarrow \infty$.

The case where $f(z, d z / d t, t) \equiv 0$, and $B_{1}(t) \equiv 0$ was first treated by Hukuwara [6]. Shorter proofs were subsequently given by Cesari [3], Weyl [15], and the author [1]. These methods are different from that used in this paper. Since the equation is linear now, it is clear that there should be no restriction upon the size of $\left\|z\left(t_{0}\right)\right\|$ or upon the magnitude of $\int_{0}^{\infty}\|B(t)\| d t$. We can always choose a $t_{0}$ sufficiertly large to make the integral $\int_{t_{0}}^{\infty}\|B(t)\| d t$ as small as desired, and once having obtained $N$ linearly independent solutions, we can use the fact that the solutions of an $N$ th order linear system constitute an $N$-dimensional linear manifold to prove that all solutions are bounded as $t \rightarrow+\infty$.

To prove the statement concerning sub-manifolds of solutions, we divide a particular set of $N$ linearly independent solutions of $d y / d t=A y$ into two subsets, one set consisting of $k$ linearly independent solutions whose norms approach 0 as $t \rightarrow+\infty$, and the other residual set consisting of solutions whose norms are merely bounded as $t \rightarrow+\infty$. These bounded solutions correspond to characteristic roots with zero real parts. A consequence of condition (1) of (4.1) is that no secular terms can occur, that is, no terms of the form $t^{k} \sin \mu t$. Thus the bounded terms are, as sums of periodic terms, almost periodic terms, and one can use the terminology almost periodic vector to describe a vector all of whose components are almost periodic functions.

The $k$-dimensional manifold of solutions whose norms approach 0 as $t \rightarrow+\infty$ is formed as in Theorem 3, except that there now need be no decomposition of $Y$.

To form the $(N-k)$-dimensional manifold we consider solutions of the integral equation

$$
\begin{aligned}
z=\sum_{r=k+1}^{N} b_{r} w_{r} & +\int_{t_{0}}^{t} Y\left(t-t_{1}\right) B\left(t_{1}\right) z d t_{1}+\int_{t_{0}}^{t} Y\left(t-t_{1}\right) B_{1}\left(t_{1}\right) \frac{d z}{d t_{1}} d t_{1} \\
& +\int_{t_{0}}^{t} Y\left(t-t_{1}\right) f\left(z, d z / d t_{1}, t_{1}\right) d t_{1}
\end{aligned}
$$

where the $w_{r}$ constitute the subset of $N-k$ linearly independent bounded solutions whose norms do not approach zero as $t \rightarrow \infty$. We assume that this subset consists of solutions all of whose components are almost-periodic 
functions. It would make no difference if some elements of the $k$-dimensional manifold were mixed in, since they will vanish for $t=\infty$. Thus we assume that they are not there to begin with.

This equation can be solved by the method of successive approximations, as usual, provided that $\sum_{r=k+1}^{N}\left|b_{r}\right|$ is sufficiently small, and we obtain the desired manifold. Since the $b_{r}$ are small, it is easy to see that this manifold can not reduce to the single element zero.

Any bounded solution of (4.3) approaches the vector function

$$
\begin{aligned}
z=\sum_{k+1}^{N} b_{r} w_{r} & +\int_{t_{0}}^{t} Y^{*}\left(t-t_{1}\right) B\left(t_{1}\right) z d t_{1}+\int_{t_{0}}^{t} Y^{*}\left(t-t_{1}\right) B_{1}\left(t_{1}\right) \frac{d z}{d t_{1}} d t_{1} \\
& +\int_{t_{0}}^{t} Y^{*}\left(t-t_{1}\right) f\left(z, d z / d t, t_{1}\right) d t_{1}
\end{aligned}
$$

where $Y^{*}$ denotes the matrix formed from $Y$ by replacing all terms corresponding to characteristic roots with negative real parts by zero. This function is almost periodic.

For the linear case, $f(z, d z / d t, t) \equiv 0, B_{1}(t) \equiv 0$, this result is due to Wintner [17], Levinson [7].

5. The case of variable $A$. If $A$ is a variable matrix, the identity $Y(t) Y^{-1}\left(t_{1}\right)=Y\left(t-t_{1}\right)$ is not valid, and there is no longer an easy way to take account of the term $Y^{-1}(t)$ appearing in Lemma 2 . If one considers this matrix directly, one obtains a matrix whose elements are cofactors of elements of $Y(t)$, divided by the scalar det $Y$, the determinant of $Y$. This determinant corresponds to the Wronskian of the $n$th order linear differential equation, and an analogous result, of which the corresponding result for the Wronskian is but a special case, can be derived, namely

$$
\operatorname{det} Y=\exp \left(\int_{0}^{t} \operatorname{trace} A d t\right) \text {. }
$$

Unless more is known about the form of the solution, as in the case where $A$ is constant, we shall be forced to use the crude estimate that each element in $Y^{-1}(t)$ is bounded by a bound depending upon the bound on the elements of $Y(t)$, multiplied by a bound for $\exp \left(-\int_{0}^{t}\right.$ trace $\left.A d t\right)$. The most important case is where trace $A=0$, in which case det $Y=1$.

More precisely, if $\exp \left(-\int_{0}^{t}\right.$ trace $\left.A d t\right)$ is to be bounded, $\int_{0}^{t}$ trace $A d t$ must be bounded away from $-\infty$, and the condition

$$
\inf \int_{0}^{t} \operatorname{trace} A d t>-\infty
$$

is a natural one. This condition was used in the linear case by Wintner [16], while the condition trace $A=0$ was used by the author [1]. The more gen- 
eral condition (5.2) was also mentioned.

Other conditions have been given by Perron [11].

If we use condition (5.2), Theorems 2 and 4 can be easily generalized.

6. Application of the Birkhoff-Kellogg fixed-point theorem. The application of fixed-point theorems of function space to the proof of the existence of solutions of differential, integral, and other types of functional equations dates from the classical paper of Birkhoff-Kellogg [2]. The scope of the method was considerably enlarged by Schauder in a series of papers, of which we mention only the last [13].

Although the fixed-point method has the advantage of furnishing existence proofs with a minimum of conditions in most cases, it compensates for this advantage in not furnishing any algorithm for obtaining the solution, and in yielding no information concerning uniqueness of the solution.

We think it worthwhile, however, to state some results obtained by this method; as illustration of how some of the previous conditions can be weakened. We shall consider only equations of the form

$$
d z / d t=A z+f(z, t) .
$$

The first case we shall consider is that where $f(z, t)$ possesses partial derivatives with respect to the $z$ which are bounded in any finite $t$-interval, but do not satisfy either condition (2) of (4.1), or condition (3) of (3.2). The existence of these bounded partial derivatives is sufficient to ensure uniqueness, and we shall combine this uniqueness with the pure existence proof obtained by means of the fixed-point theorem to show that under conditions similar to those discussed in the previous sections, all solutions of (6.1) will be bounded.

The second case treated is where $f(z, t)$ satisfies the condition $\|f(z, t)\|$ $=o(\|z\|)$, or $o(\|z\| h(t)), h(t)$ integrable over $(0, \infty)$, as $\|z\| \rightarrow 0$. Here we shall use a method which seems due to Hukuwara [6] to extend the fixed-point theorem from the finite interval to the infinite interval.

When (6.1) is converted into an integral equation by means of Lemma 2, we obtain the equation

$$
z=y+\int_{0}^{\ell} Y\left(t-t_{1}\right) f\left(z, t_{1}\right) d t_{1}=T(z)
$$

where we use $T(z)$ to represent a functional transformation. The existence of a solution of (6.2) is equivalent to the existence of a fixed-point of the transformation $T(z)$, that is, a function satisfying the equation $z=T(z)$. Under certain conditions, which we shall discuss below, we can assert the existence of such a fixed-point. This is the substance of the following theorem due to Birkhoff-Kellogg, which we state as a lemma.

Lemma 3. Let $R$ denote the totality of real vector functions, $f(t)$, definei 
over a finite, closed interval $\left(t_{0}, t_{1}\right)$, which have the property that $\left\|f\left(t_{1}\right)-f\left(t_{2}\right)\right\|$ $\leqq c_{1} \epsilon$ whenever. $\left|t_{1}-t_{2}\right| \leqq \epsilon$, and which are uniformly bounded by $c_{2}$. Let $\cdot S(f)$ denote a one-valued continuous transformation which carries each point of $R$ into a point of $R$.

Then there exists a point, $f(t)$, of $R$ which is invariant under the transformation, that is, $S(f)=f$.

In order to apply this lemma, it is necessary to state sets of conditions under which $T(z)$ will satisfy the conditions of the lemma. There is no difficulty verifying that, provided $\|y\|$ is sufficiently small, and either set of the following conditions is satisfied, $T(z)$ is a transformation of the desired type.

(6.3) (1) $\int_{0}^{\infty}\|Y(t)\| d t<\infty$.

(2) $\|f(z, t)\|=o(\|z\|)$ as $\|z\| \rightarrow 0$, uniformly in $t$.

(3) $f(z, t)$ is a continuous function of $z$.

(6.4) (1) $\|Y(t)\| \leqq c_{3}, t \geqq 0$.

(2) $\|f(z, t)\|=o(\|z\| h(t))$, as $\|z\| \rightarrow 0$.

(3) $\int_{0}^{\infty} h(t) d t<\infty$.

(4) $f(z, t)$ is a continuous function of $z$.

Applying Lemma 3, we shall obtain the following two results, which are improvements of Theorems 2 and 4 respectively.

THEOREM 5. Consider the differential equation of (6.1), where:

(6.5) (1) All the characteristic roots of $A$ have negative real parts.

(2) Conditions (2), (3) of (6.3) are satisfied.

(3) The partial derivatives $f_{z_{k}}(z, t)$ exist, and are uniformly bounded in any finite t-interval.

Under these conditions, the norm of every solution, $z$, for which the norm of the initial value, $\|z(0)\|$, is sufficiently small, approaches 0 as $t \rightarrow 0$.

THEOREM 6. Consider the differential equation of (6.1) where:

(6.6) (1) $A$ is a constant matrix and all solutions of $d y / d t=A y$ are bounded.

(2) Conditions (2), (3), (4) of (6.4) are satisfied.

(3) The partial derivatives $f_{z_{k}}(z, t)$ exist, and are uniformly bounded in any finite t-interval.

Under these conditions, the norm of every solution, $z$, for which the norm of the initial value, $\|z(0)\|$, is sufficiently small is uniformly bounded for all $t$.

Proof of Theorems 5 and 6 . Converting the differential equation of (6.1) into the integral equation of (6.2) we apply Lemma 3 for the finite intervals $\left(0, t_{1}\right),\left(0, t_{2}\right)$. For each finite interval there exists a solution. The bound on each solution is the same, since it can be taken to be $2 \max \|y\|, t \geqq 0$. Furthermore, each solution is equal to $y(0)$ at $t=0$. Thus, using the uniqueness theorem, Lemma 2, the two solutions must be identical over the common interval of definition. Thus the solution is uniformly bounded over any 
$t$-interval. The method of proving that $\|z\| \rightarrow 0$ in Theorem 5 will be illustrated in the proof of Theorem 7 below.

It is easy to construct examples of functions, $f(z, t)$, satisfying the conditions of Theorems 5 and 6 , but not those of Theorems 2 and 4. Relative to Theorem 5 , we might take the function which has a component such as $z_{1}^{2} \sin z_{1} e^{t}$, and for Theorem 6 , we can use the function $z_{1}^{2} \sin z_{1} t /\left(t^{2}+1\right)$.

To treat the case where the partial derivatives do not exist, we require the following lemma.

LEMma 4. Let $T(f)$ be a continuous transformation which converts functions which are bounded and continuous over the infinite interval $(0, \infty)$ into functions which are bounded over the infinite interval, with the same bound, provided that this bound is small enough, and equi-continuous over any finite sub-interval. Then the equation $T(f)=f$ has a solution.

Proof. Consider a sequence of increasing intervals, $(0, n), n=1,2, \cdots$. Take the class of functions which are continuous and uniformly bounded over this interval, where the bound is small enough so that the bound of the transform is the same. Applying Lemma 3, we see that there is a function defined over the interval $(0, n)$ which satisfies the equation $T(f)=f$. Let this function be $f_{n}(t)$, and define it for $t \geqq n$ by $f_{n}(t)=f_{n}(n)$. The new function is then continuous for all $t$, and uniformly bounded. Consider the sequence of functions, $f_{1}(t), f_{2}(t), \cdots, f_{n}(t), \cdots$. The sequence has a uniform bound for all its terms and, by hypothesis, is equi-continuous over any finite interval. Using the Arzela selection theorem, we can choose a subsequence which converges uniformly over the interval $(0,1)$. Considering the interval $(0,2)$ we can choose a subsequence of this subsequence which converges uniformly over this interval. Continuing in this way, and then using the familiar diagonal process, we obtain a sequence which converges everywhere, and uniformly in every finite $t$-interval. Call this sequence $\left(f_{n}^{*}(t)\right)$, and the limit function $f^{*}(t)$. We now wish to show that $f^{*}(t)$ is the desired solution. For any finite $t$, we have, for $n$ large enough, $T\left(f_{n}^{*}(t)\right)=f_{n}^{*}(t)$. Now if we let $n \rightarrow \infty$, $T\left(f_{n}^{*}\right) \rightarrow T\left(f^{*}\right)$, since $T(f)$ is a continuous transformation, and the sequence $\left(f_{n}^{*}(t)\right)$ converges uniformly in any finite interval. Thus $T\left(f^{*}\right)=f^{*}$.

The transformation, $T(z)$, of (6.2), satisfies the conditions of Lemma 4 , and thus we can improve Theorems 2 and 4 still further, and can now state the following theorem.

TheOREM 7. Consider the differential equation of (6.1), where:

(6.7) (1) All the characteristic roots of $A$ have negative real parts.

(2) $\|f(z, t)\|=o(\|z\|)$ as $\|z\| \rightarrow 0$, uniformly in $t$.

(3) $f(z, t)$ is a continuous function of $z$.

Under these conditions, there exists an $N$-dimensional manifold of solutions whose norms approach 0 as $t \rightarrow \infty$. 
TheOREM 8. Consider the differential equation of (6.1), where:

(6.8) (1) $A$ is a constant matrix, and all solutions of $d y / d t=A y$ are bounded.

(2) $\|f(z, t)\|=o(\|z\| h(t))$, as $\|z\| \rightarrow 0$.

(3) $\int_{0}^{\infty} h(t) d t<\infty$.

(4) $f(z, t)$ is a continuous function of $z$.

Under these conditions there exists an $N$-dimensional manifold of bounded solutions.

Proof. To obtain Theorem 7 in its full form, with the statement concerning the norm approaching 0 , we have to make a slight change of variable. Let $z=w e^{-\lambda t}$, where $\lambda>0$, and less than the minimum of the absolute values of the real parts of the characteristic roots. The equation of (6.2) becomes

$$
w=y e^{-\lambda t}+e^{\lambda t} \int_{0}^{t} Y\left(t-t_{1}\right) f\left(w e^{-\lambda t_{1}}, t_{1}\right) d t_{1}=T(w) .
$$

$T(w)$ enjoys the same properties as did $T(z)$, and a bounded solution of $T(w)=w$ leads to a solution of (6.2) satisfying the condition $\|z\| \leqq c_{4} e^{-\lambda t}$. To obtain Theorem 8, Lemma 4 is applied directly to $T(z)$.

We use this method to extend Theorem 3. The transformation in this case is given by (cf. (3.21))

$$
S(z)=y+\int_{0}^{t} Y_{1}\left(t-t_{1}\right) f\left(z, t_{1}\right) d t_{1}-\int_{t}^{\infty} Y_{2}\left(t-t_{1}\right) f\left(z, t_{1}\right) d t_{1} .
$$

Performing the substitution $z=w e^{-\lambda t}$, where $\lambda>0$ and less than the minimum of the real parts of the characteristic roots with negative real parts, we obtain a transformation satisfying the conditions of Lemma 4, and thus we have the following extension of Theorem 3.

TheOREM 9. Consider the differential equation of (6.1) where:

(6.11) (1) $k$ of the characteristic roots of $A$ have negative real parts.

(2) $\|f(z, t)\|=o(\|z\|)$ as $\|z\| \rightarrow 0$, uniformly in $t$.

(3) $f(z, t)$ is a continuous function of $t$.

Under these conditions there exists a $k$-dimensional manifold of solutions whose norms approach 0 as $t \rightarrow \infty$.

Similar results can be obtained for the case of $A$ a variable matrix, under the conditions upon trace $A$ specified previously, but the results do not seem comprehensive enough to warrant quoting.

\section{Part II. Difference equations}

7. Introductory remarks. The problem generally considered in the modern theory of difference equations is the investigation of analytic solutions of equations of the form 


$$
\begin{aligned}
x_{i}(t+1)=F\left(x_{1}(t), x_{2}(t)\right. & \left., \cdots, x_{N}(t), t\right), \\
i & =1,2, \cdots, N ;-\infty<t<\infty .
\end{aligned}
$$

On this subject there exists a very extensive literature, as may be seen by reference to N $\phi$ rlund's treatise [9]. In practice, one has often to deal with a type of difference equation, or recurrence relation, as it is frequently called, of the type

$$
\begin{aligned}
x_{i}(t+1)=F\left(x_{1}(t), x_{2}(t), \cdots,\right. & \left.x_{N}(t+1), t\right), \\
& i=1,2, \cdots, N ; t=0,1, \cdots .
\end{aligned}
$$

Here the question is usually to find an explicit expression for $u_{i}(t)$ in terms of the initial values, $u_{i}(0)$, and $t$. If the system is complicated, and in particular, if it is nonlinear, it may be very difficult, or even impossible, to find an explicit expression.

There still remains the very interesting possibility of discussing the asymptotic behavior of the solution for increasing $t$, or at least of obtaining upper bounds for the functions $u_{i}(t)$. As we shall see, one finds here a quite complete parallel to the corresponding problem for differential equations discussed in the first part.

$\mathrm{Ta} \mathrm{Li}$ [14] obtained, for difference equations of the form (7.2), analogues of the results of Perron [11]. In the linear case, Ford [4, 5], applying a method of Dini's developed for use in differential equations, obtained results analogous to Theorem 4.

We intend, in this section, to generalize and extend the results of $\mathrm{Ta} \mathrm{Li}$ and Perron, and incidentally to furnish simpler proofs of some of their results. Then, as an application of these results, we shall sketch an alternate proof of Theorems 5, 6, 7, 8, 9.

8. Preliminaries. Consider the difference equations

$$
\begin{aligned}
\Delta z_{i} & =z_{i}(t+1)-z_{i}(t)=\sum_{j=1}^{N} a_{i j}(t) z_{i}+f_{i}\left(z_{1}, z_{2}, \cdots, z_{N}, t\right), \\
\Delta z_{i} & =\sum_{j=1}^{N} a_{i j}(t) y_{j}, \quad i=1,2, \cdots, N ; t=1,2, \cdots,
\end{aligned}
$$

where, as customary, we are interested in the behavior of the solutions for large values of $t$, and the relations between the solutions of the two equations. The results will be similar to those obtained for differential equations, and the proofs even simpler.

We shall confine our attention to systems and the notation shall be as for differential equations. Equations (8.1) and (8.2) can be written

$$
\begin{aligned}
\Delta z & =A z+f(z, t), \\
\Delta y & =A y .
\end{aligned}
$$


Usually the elements of $A$ will be independent of $t$. In that case, as before, we say that $A$ is a constant matrix. If the elements depend upon $t$, it will be supposed that they are defined for every $t$ in the range $(0, \infty)$.

It is important to note that we can derive similar results if we consider $t$ to be a continuous variable over the range $(0, \infty)$. A general solution of $(8.3)$ can be defined, with periodic functions, of period 1 , replacing the constants appearing in differential equations. To obtain the behavior of the solution for any particular large value of $t$, one has to reduce $t$ modulo 1 , until one finds the point in the interval $(0,1)$ which could have been used as a zeropoint to obtain $t$. If one has a solution of (8.4) with certain uniform properties one can deduce corresponding properties of the solution of (8.3). It seems simpler to state the results for the recurrence relations treated here. It is worth noting that they could be transliterated in terms of ordinary difference equations.

The uniqueness theorem is now completely on the surface, but we mention it here for future reference:

Lemma 5. If $y(t)$ and $z(t)$ are two solutions of

$$
z(t+1)=F(z(t), t)
$$

which are equal at $t=t_{0}$, they are equal for all larger values of $t$, provided that $F(z, t)$ is $a$ one-valued function of $z$ and $t$.

Nothing is said about the relationship between $y$ and $z$ for smaller values of $t$. In general, nothing can be said, for although (8.5) yields $z(t+1)$ in terms of $z(t)$, the reverse is not necessarily true, if we ask for one-valued functions. Fortunately, small values of $t$ are of no interest to us.

If $A$ is a constant matrix, the solution of $\Delta y=A y$ is determined by the characteristic roots of $A+I$. Instead of the trial solution $c e^{\lambda t}$ used in differential equations, one uses here $c \lambda^{t}$. Eliminating the components of $c$, one obtains the characteristic equation $|A+I(1-\lambda)|=0$.

Thus the solutions of $\Delta y=A y$ will consist of the real and imaginary parts of $P(n)(\lambda+i \mu)^{\imath}$, the polynomials $P(n)$ appearing if the characteristic matrix has multiple elementary divisors. As in the case of differential equations, the behavior at infinity is determined by the nature of the roots of the characteristic equation. There are crucial regions, inside the unit circle, and outside the unit circle, with the circumference of the circle furnishing a third type of solution. Roots lying inside the circle furnish manifolds of solutions which approach 0 as $t \rightarrow \infty$, roots outside the circle furnish manifolds of solutions which approach $\infty$ as $t \rightarrow \infty$; roots on the circumference furnish manifolds of oscillating solutions.

The vector equation, (8.4), has $N$ linearly independent solutions, $y^{(1)}, y^{(2)}, \cdots, y^{(N)}$. These $N$ linearly independent solutions can be chosen so that the $N \times N$ matrix whose columns are the $y^{(k)}$ satisfies the initial condi- 
tion $Y(0)=I$. It is clear that $Y$ satisfies the matrix equation $\Delta Y=A Y$.

The basic method of this section is the analogue of the basic method of the first part of the paper, namely conversion of the difference equation into a sum equation. This sum equation is the analogue of the integral equation used in the first part. The procedure is discussed in the following lemma.

LEMMA $6\left({ }^{2}\right)$. The solution of

$$
\Delta z=A z+w, \quad z\left(t_{0}\right)=y\left(t_{0}\right),
$$

where $y$ is a solution of (8.4), is

$$
z(t)=y(t)+\sum_{t_{1}=t_{0}}^{t-1} Y(t) Y^{-1}\left(t_{1}+1\right) w\left(t_{1}\right) .
$$

If $A$ is a constant matrix, this reduces to

$$
z(t)=y(t)+\sum_{t_{1}=t_{0}}^{t-1} Y\left(t-t_{1}-1\right) w\left(t_{1}\right) .
$$

Proof. The proof is again by means of variation of parameters. Let $z=Y u$. Then

$$
\begin{aligned}
\Delta z & =Y(t+1) u(t+1)-Y(t) u(t) \\
& =(Y(t+1)-Y(t)) u(t)+Y(t+1)(u(t+1)-u(t)) \\
& =(\Delta Y(t)) u(t)+Y(t+1) \Delta u(t) .
\end{aligned}
$$

Thus

$$
\begin{aligned}
(\Delta Y(t)) u(t)+Y(t+1) \Delta u(t) & =A Y(t) u(t)+w(t), \\
\Delta u(t) & =Y^{-1}(t+1) w(t),
\end{aligned}
$$

and finally

$$
u=v+\sum_{t_{1}=t_{0}}^{t-1} Y\left(t_{1}+1\right) w\left(t_{1}\right),
$$

where $v$ is the first column of $I$, the identity matrix. Thus

$$
z=y+\sum_{t_{1}=t_{0}}^{t-1} Y(t) Y^{-1}\left(t_{1}+1\right) w\left(t_{1}\right) .
$$

The notation requires a note of explanation. The vacuous sum $\sum_{t_{1}=t_{0}}^{t_{0}-1}$ will be taken to be zero. The sum $\sum_{t_{1}-t_{0}}^{t_{0}}$ will be understood to represent the summand at $t=t_{0}$. Expressions of this type will be used only where $t \geqq t_{0}$.

(2) Lemma 6 is valid if we add the condition that $A+I$ is nonsingular for $t=0,1,2, \ldots$, and hence $Y$ is also nonsingular for $t=0,1,2, \ldots$ If $A$ is a constant matrix, this means that $A$ has no zero characteristic roots. 
If $A$ is a constant, $Y(t) Y^{-1}(1)$ and $Y(t-1)$ both represent solutions of $\Delta Y=A Y$ which have the same initial value at $t=1$, and therefore must be equal for $t \geqq 1$. In this way, by induction, one can prove the general inequality $Y(t) Y^{-1}\left(t_{1}\right)=Y\left(t-t_{1}\right)$.

9. The matrix $A$ is constant. In this section we present the result corresponding to Theorems 1 and 4 . We treat the simple case first, where $z(t+1)$ does not occur on the right-hand side, and we shall indicate how also to handle this equation.

Theorem 10. Consider the difference equation

$$
\Delta z=(A+B) z+f(z, t),
$$

where either of the following sets of conditions is satisfied:

(9.2) (1) The characteristic roots of $A+I$ all lie inside the unit circle.

(2) $\|B\| \leqq c_{1}, c_{1}$ depending upon $A$ and $f(z, t)$.

(3) $\|f(z, t)\|=o(\|z\|)$, as $\|z\| \rightarrow 0$, uniformly in $t$.

(9.3) (1) $A$ is a constant matrix, and all solutions of $\Delta y=A y$ are bounded as $t \rightarrow \infty$.

(2) $\sum_{t=0}^{\infty}\|B(t)\| \leqq c_{1}, c_{1}$ depending upon $A$ and $f(z, t)$.

(3) $\|f(z, t)\|=a(h(t)\|z\|)$, and $\sum_{t=0}^{\infty} h(t)<\infty$, as $\|z\| \rightarrow 0$.

If conditions (2.2) are satisfied, every solution, $z$, of (9.1) for which the norm of $z(0)$ is sufficiently small approaches 0 as $t \rightarrow \infty$.

If conditions (9.3) are satisfied, every solution, $z$, of (9.1) for which the norm of $z(0)$ is sufficiently small is uniformly bounded as $t \rightarrow \infty$.

Proof. Using Lemma 6, convert (9.1) into the sum-equation

(9.4) $z=y+\sum_{t_{1}=0}^{t-1} Y\left(t-t_{1}-1\right) B\left(t_{1}\right) z\left(t_{1}\right)+\sum_{t_{1}=0}^{t-1} Y\left(t-t_{1}-1\right) f\left(z, t_{1}\right)$

where $y$ is the solution of (8.4) with the same initial value as $z$, and $Y$ is the matrix solution of $\Delta Y=A Y$ with the initial value $Y(0)=I$. Using the same reasoning as applied in Theorems 2 and 4 , it is easy to show by induction that one has uniform bounds on the solutions. The proof of the statement concerning the norms tending to zero is proved by means of a suitable substitution, as in Theorem 7. It is easy to see that we can obtain the analogue of Theorem 3 using the same decomposition as employed there.

10. $A$ is a variable matrix. The same difficulties that arose in the case of differential equations can be partially overcome here also by an appropriate restriction upon the trace of $A$. Again the case trace $A=0$ is the most important.

It is easy to verify that the determinant of $Y$, det $Y$, satisfies the difference equation

$$
\Delta W=(\operatorname{trace} A) W, \quad W(0)=1,
$$


and thus

$$
\operatorname{det} Y=\prod_{t_{1}=0}^{t-1}\left(1+\operatorname{trace} A\left(t_{1}\right)\right), \quad t \geqq 1(\mathcal{z}) .
$$

Thus, results similar to those for differential equations may be stated.

11. The case where $z(t+1)$ appears on the right-hand side. Considering the more general equation of the form

$$
z(t+1)-z(t)=A z(t)+f(z(t), z(t+1), t),
$$

we may still use Lemma 6 to convert it into the form of (9.4). Here, however, the right side will contain $z(t)$, and to obtain a solution we may seek recourse to the method of successive approximations, putting appropriate conditions on the partial derivatives of the $f(z(t), z(t+1), t)$ to ensure convergence, or we may use an algebraic form of the fixed-point theorem. The equation does not seem of enough interest to warrant detailed discussion, and thus we shall be content with these few remarks.

12. Application to differential equations. To apply some of the above results to differential equations, we consider the differential equation

$$
d z / d t=A z+f(z, t),
$$$$
t \geqq 0,
$$

as the limiting case of difference equations of the form

$$
z(t+h)=(I+A h) z(t)+h f(z, t), \quad t=0, h, 2 h, \cdots ; h>0,
$$

or

$$
z(t+h)-z(t)=h A z(t)+h f(z, t), \quad t=0, h, 2 h, \cdots .
$$

We discuss first the case where all the characteristic roots of $A$ have negative real parts. The equation of first approximation of (12.2) is

$$
y(t+h)=(I+h A) y(t), \quad t=0, h, 2 h, \cdots .
$$

The trial solution $y(t)=c \alpha^{t}$ yields the characteristic equation

$$
\left|A-I\left(\frac{\alpha^{h}-1}{h}\right)\right|=0 .
$$

Comparing this with the characteristic equation $|A-\lambda I|=0$, we see that to each $\lambda$ there corresponds one or more $\alpha$ given by

$$
\frac{\alpha^{h}-1}{h}=\lambda, \quad \alpha^{h}=1+h \lambda .
$$

Expressing $\lambda$ in the form $\lambda_{1}+i \mu_{1}$, where $\lambda_{1}<0$, we see that

(3) These equations are incorrect. The author was misled by the corresponding results for differential equations. 


$$
|\alpha|=\left[\left(1+h \lambda_{1}\right)^{2}+h^{2} \mu_{1}^{2}\right]^{1 / 2}=\left[1+2 h \lambda_{1}+h^{2}\left(\lambda_{1}^{2}+\mu_{1}^{2}\right)\right]^{1 / 2 h} .
$$

As $h \rightarrow 0, \lim |\alpha|=e^{\lambda_{1}}$. Thus for $h$ less than a given positive number, all the $\alpha$ will be less than 1 in absolute value. Furthermore, let $r e^{i \theta}=1+h \lambda$, $r>0,-\pi \leqq \theta \leqq+\pi$, and choose $\alpha=r^{1 / h} e^{i \theta / h}$. It follows then that $\lim \alpha=e^{\lambda}$.

The solution of (12.2) for which $z(0)=y(0)$ can be written

$$
z(t)=y(t)+h \sum_{t_{1}=0}^{t-h} Y\left(t-t_{1}-h\right) f\left(z, t_{1}\right), \quad t=0, h, 2 h, \cdots .
$$

Choosing $h$ small enough, we see that, under the condition the characteristic roots of $A$ all have negative real parts, the matrix $Y(t)$, which is the solution of $Y(t+h)=(I+h A) Y(t)$, is uniformly bounded in $t$ and $h$.

Choose $y_{0}$, the initial value, to be independent of $h$. Then $y(t)$, the solution of (12.4), satisfying $y(0)=y_{0}$, is given by $y=Y(t) y_{0}$, and thus $\|y(t)\|$ $\leqq\|Y(t)\|\left\|y_{0}\right\| \leqq c_{1}\left\|y_{0}\right\| \leqq c_{2}$ for all $t$, and $0 \leqq h \leqq h_{1}$. For $h=0$, it is clear that the solutions of (12.4) go over into the solutions of $d y / d t=A y$.

We have $Y(n h)=Y(h)^{n}$. Thus, since $\|Y(h)\| \leqq c_{4} c_{3}^{n}, 0<c_{3}<1$, we have

$$
h \sum_{n=0}^{\infty}\|Y(n h)\| \leqq c_{4} h \sum_{n=0}^{\infty} c_{3}^{n h}=c_{4} h /\left(1-c_{3}\right) \leqq c_{4} /\left(\log 1 / c_{3}\right)=c_{5},
$$

for small $h$.

We now wish to show that the $z(t)$ are uniformly bounded in $t$ and $h$ if we impose the following familiar condition upon $f(z, t)$,

$$
\|f(z, t)\| \leqq c_{6}\|z\|
$$

as $\|z\| \rightarrow 0$ uniformly in $t$,

where $c$ is a suitable small constant which will be chosen later.

We note that all previous results could have been expressed using a condition of this sort, instead of considering separately a $B z$ term, and a term satisfying the condition $o(\|z\|)$ as $\|z\| \rightarrow 0$, uniformly in $t$. We preferred to use this type of condition since it seemed to exhibit the role of the nonlinearity very clearly.

Let us assume that $\|z(k h)\| \leqq 2 c_{2}$, for $k=0,1,2, \cdots, n$, and let us show that this implies the same for $z((n+1) h)$. We have, using (12.8),

$$
\begin{aligned}
z\|((n+1) h)\| & \leqq\|y\|+h \sum_{t_{1}=0}^{n h}\left\|Y\left(t-t_{1}-h\right)\right\|\left\|f\left(z, t_{1}\right)\right\| \\
& \leqq c_{2}+2 c_{2} c_{6} h \sum_{t_{1}=0}^{n h}\left\|Y\left(t-t_{1}-h\right)\right\| \\
& \leqq c_{2}+2 c_{2} c_{8} h \sum_{n=0}^{\infty}\|Y(n h)\| \leqq c_{2}+2 c_{2} c_{5} c_{8} \leqq 2 c_{2},
\end{aligned}
$$

provided that $c_{6}$ is small enough. 
Thus, for each positive $h$, sufficiently small, we obtain a function, $z_{h}(t)$, defined for $t=0, h, 2 h, \cdots$, satisfying (12.3), uniformly bounded in $t$ and $h$. We can define these functions for all $t$ by requiring that they be linear in the intervals $(k h,(k+1) h), k=0,1,2, \cdots$. Thus defined they will be continuous for all $t \geqq 0$. Let us now choose a particular sequence of $h$ values, $h, h / 2$, $h / 4, \cdots, h / 2^{n}, \cdots$. We now wish to prove that the corresponding sequence of functions, solutions of (12.3) for these different $h$ 's, is equicontinuous as well.

Using the difference equation (12.3) we see that

$$
\begin{aligned}
\|z(t+h)-z(t)\| & \leqq h\|A\|\|z\|+h\|f(z, t)\| \\
& \leqq h\|A\|\left(2 c_{2}\right)+h\left(2 c_{2}\right) c_{0} \leqq c_{7} h .
\end{aligned}
$$

This combined with the linearity in the intervals $(k h,(k+1) h)$ yields the required equicontinuity.

We now choose any finite interval, $\left(0, c_{8}\right)$, and apply the Arzela selection theorem to the sequence of uniformly bounded equicontinuous functions. This yields a uniformly convergent sequence. Double the interval and repeat the process with the new sequence. Continuing in this manner and using the diagonal process, we obtain a sequence, which we designate by $z_{1}, z_{2}, \cdots$, $z_{n}, \cdots$, which converges for all $t$ and uniformly in any finite interval.

Instead of attempting to prove uniform differentiability properties of this sequence, and thus show that the limit function satisfies the differential equation of (12.1), we use the relationship of (12.8) to show that the limit function, $z(t)$, satisfies the integral equation

$$
z(t)=y(t)+\int_{0}^{t} Y\left(t-t_{1}\right) f\left(z, t_{1}\right) d t_{1} .
$$

The proof of this is akin to the proof of the existence of the Riemann integral, and with the weapon of uniform convergence for any finite interval the proof presents no difficulties. We thus omit it. From (12.13) it follows that $z(t)$ is differentiable and satisfies (12.1).

We have thus outlined another proof of Theorem 7, using much less sophisticated methods than the fixed-point theorem for function spaces. Similarly, we can prove Theorem 9 . Theorem 8 presents a slight difficulty, since zero real parts may occur. Returning to equation (12.7), we have in that case

$$
|\alpha| \leqq\left[1+h^{2} \mu_{1}^{2}\right]^{1 / 2 \hbar} \leqq e^{\mu_{1}^{2} h} .
$$

Thus $\alpha^{t}$ will be uniformly bounded in an interval of length $c_{8} / h$. As $h \rightarrow 0$, the interval increases, and we can always use the subsequence method to obtain a sequence converging uniformly in any finite interval, and with uniform bound over the infinite interval for the limit function. 


\section{REFERENCES}

1. R. Bellman, The stability of solutions of linear differential equations, Duke Math. J. vol. 10 (1943) pp. 643-647.

2. G. D. Birkhoff, and O. D. Kellogg, Invariant points in function space, Trans. Amer. Math. Soc. vol. 23 (1922) pp. 95-115.

3. L. Cesari, Un nuovo criterio di stabilita per le soluzione della equazioni differenziali lineari, Annali Scuola Normale Superiore Pisa (2) vol. 9 (1940) pp. 163-186.

4. L. Ford, Sur les equations linéaires aux differences finies, Annali di Matematica vol. 13 (1907) pp. 263-328.

5. - On the integration of homogeneous linear difference equations of second order, Trans. Amer. Math. Soc. vol. 10 (1909) pp. 319-336.

6. M. Hukuwara, Sur les points singuliers des equations differentielles lineaires, Journal of the Faculty of Science, Hokkaido Imperial University, Series I, Mathematics vol. 2 (19341936) pp. 13-88.

7. N. Levinson, The asymptotic behavior of a system of linear differential equations, Amer. J. Math. vol. 68 (1946) pp. 1-6.

8. A. Liapounoff, Probleme géneral de la stabilité du mouvement, Ann. Fac. Sci. Univ. Toulouse (2) vol. 9 (1907) pp. 203-475.

9. N. Nфrlund, Differenzrechnung, Berlin, 1924.

10. O. Perron, Uber Stabilitat und asymptotisches verhalten der Integrale von Differentialgleichungssytemen, Math. Zeit. vol. 29 (1929) pp. 129-160.

11. - Die Stabilitutsfrage bei Differentialgleichungen, Math. Zeit. vol. 32 (1930).

12. - Uber Stabilitat und asymptotisches verhalten der Lösungen eines Systemes endlicher Differenzengleichungen, J. Reine Angew. Math. vol. 161 (1929).

13. J. Schauder, Die Fixpunktsatz in Funktionalraum, Studia Mathematica vol. 2 (1930) pp. 171-181. 141.

14. Ta Li, Die Stabilitatsfrage bei Differenzgleichungen, Acta Math. vol. 63 (1934) pp. 99-

15. H. Weyl, Remark on the preceding paper, Amer. J. Math. vol. 68 (1946) pp. 7-13.

16. A. Wintner, Linear variations of constants, Amer. J. Math. vol. 68 (1946) pp. 185-213.

17. - Small perturbations, Amer. J. Math. vol. 67 (1945) pp. 417-430.

18. H. Poincaré, Les méthodes nouvelles de la mécanique cêleste, Paris, 1892.

Princeton University, PRINCETON, N. J. 